\title{
COMMENTS ON THE DEBIJE-BRINKMAN EQUATION
}

\author{
F W WIEGEL and P F MIJNLIEFF \\ Department of Applied Physics, Twente Universtiy of Technology, \\ Enschede, The Netherlands
}

Received 3 March 1976

\begin{abstract}
We present a macroscopic derivation of the Debije-Brinkman equation for the flow of a fluid through a polymer coil By using an exact relation between the sedimentation coefficient and the permeability it is found that the permeability of the coil is strongly dependent on the nature of the fludd, due to local clustering of the polymer segments
\end{abstract}

The hydrodynamics of a dilute polymer solution has been the subject of many investugations These ultımately aim at expressing the transport coefficients of the solution in terms of the molecular weight and chemical composition of the polymer and the interaction between polymer and solvent Debıje ${ }^{1}$ ) and Brınk$\operatorname{man}^{2}$ ) were the first to postulate that the flow of the solvent is determined in the stationary state by the equation

$$
-\Gamma P+\eta_{0} J \boldsymbol{V}-\left(\eta_{0} i k\right)(V-U)=0,
$$

which we call the Debıje-Brinkman equation, and by the relation

$$
\operatorname{d} ı v V=0 \text {, }
$$

which expresses the incompressibility of the solvent In these equations $\boldsymbol{V}, \boldsymbol{U}$ and $P$ denote the local velocity of solvent and solute and the local pressure The viscosity of the pure solvent is denoted by $\%$, and the local permeability by $k$

Operatıonally the permeability is defined by an experıment in which the solvent flow's through a porous plug (a medium containıng homogeneously distributed material in fixed positions) with a constant velocity $V$ The pressure gradient needed to accomplish this situation is given by Darcy's lail

$$
-\nabla P=\left(\eta_{0} / k\right) V
$$


Recently Felderhof and Deutch ${ }^{3}$ ) and Felderhof ${ }^{+}$, presented a microscopic derixation of an equation seri smilar to eq (1), and calculated the mos important IIansport coefficients Their work ma! be considered as an extension of the micioscopic treatment of Kirkwood and Riseman Representing the polymes br a cloud of segments with number density $(0(1)$ and assuming a friction constant : per segment. Felderhof and Deutch derive an equation which is identical to the Debije-Brinkman equation provided the peimeability is related to the segment densily by

$$
1, k=-!
$$

Howeve, this form of the Debije-Brinkman equation still contans the unknow $n$ microscopic quantity $\leftarrow$ ! and is, therefore from a practical point of iell not yet an adequate startıng point for the quantıtatıse calculation of the transport coefficient, Actually, the a priori calculation of iefrom the moleculat constitution of solute and solvent is d difficult and at present unsolved problem

In this note we present a macroscopic derwation of the Debije-Brinkman equation ( 1 ) and we give an exact relation between the permeabilıty and the sedımentation coefficient In this way the equation is written in terms of the sedinentation coefficient, which can be measured accurately

The sistem considered is a mixture of the solvent (mass density $C_{0}$ ) and solute (mass density $C_{1}$ ) These two local densities $C_{0}(\boldsymbol{r})$ and $C_{1}(\boldsymbol{r})$ are macroscopic densities, $l t$ they are arerages of the corresponding microscopic densities over

1) a spatial region the linear dimension of which $(L)$ is large compared to the size of a repeatıng unit of a polymer, but small compared to the diameter of a polymer,

2) a time inteival the length of which $(T)$ is large compared to the relaxation time of part of the polymer chain enclosed in a iolume $L^{3}$, but small compared to a characteristic time of the imposed motion

The microscopic velocity $u(r)$ and pressure $p(\boldsymbol{r})$ of the fluid form that solution of the Navier-Stokes equation

$$
\left.-\Gamma p+i_{0}\right\rfloor v=0
$$

which fulfills the many boundary conditions on the particles and the walls of the container (here we considered the statıonary state and neglected the inertial term) The macroscopic velocity $V(\boldsymbol{r})$ and pressure $P(\boldsymbol{r})$ can be obtaıned by averaging $\boldsymbol{v}(\boldsymbol{r})$ and $p(\boldsymbol{r})$ over the space-tıme domains $L^{3} T$ discussed before For these macroscopic fields the Navier-Stokes equation will hold in its usual form

$$
-\Gamma P+\mu_{0} \nabla V+F=0
$$

where $\boldsymbol{F}$ denotes the external force exerted on the solvent per unit volume 
In the ease under consideration this tolce is onls due to the solute $10 \quad \Gamma$ has to be identified with the total force which the solute exests on the solvent pet unit volume Hence $\boldsymbol{F}$ could more appropriately be called a qual esternal force the worcep of quasi external forces 1 not at all new Mote than fitts years ago alreads

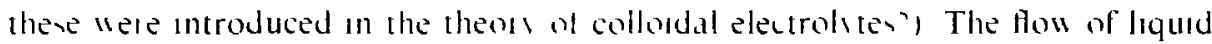
around a relected charged paltacle was dexubed bi means of ey $(6)$ with $F$ ieplaced his $E$ the product of the chatge densta of the hquad and the electiostatio field vicngith

The lince $\boldsymbol{F}$ whult the solute evelts on the olent pes unt wolume can be alculated in the following "as Fusth thrs force "ill depend onls on the relatre selocis il $-L$ f of the solvent "ith tespect to the solute Hence one can calculate $F$ for a stuation in which the solute is at tert and m which the solvent has a

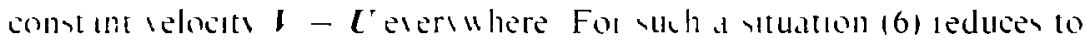

$$
-\Gamma P_{1}+\boldsymbol{F}=0
$$

But in this case Darcs s lail eq (3) gllen

$$
-\Gamma P_{0}=(1, h)\left(V^{\prime}-C^{\prime}\right)
$$

Combination of the last two equations gives the expression

$$
F=-(1 / 0 h)\left(I-C^{\prime}\right)
$$

for the force eserted on the solvent pei unit volume 1 hen this result is subst1tuted into (6) one finds the Debije-Brinkman equation (1)

Finally we would lıke to remark that the permeabilit can be expressed in terms of the sedimentation coefficient $s$ by the iclation

$$
\frac{\prime \prime 0}{h}=\frac{C_{1}}{s}\left(1-\frac{v_{1}}{v_{0}}\right) C_{0} l_{0}
$$

Here $\iota_{0}$ denotes the volume of a unit mass of the solvent and $v_{1}$ the solume of a unit mass of polymeric material $4 s C_{1} \ll 1$ the product $C_{0} l_{0}$ is practically equal to unity and can be omitted $A$ derisation of $(10)$ by using the formalism of irreversible thermodynamics can be found in a paper by Mijnlieff and Jaspers ${ }^{6}$ ) This relation is very useful, because for the systems considered sedimentation coeficients can be determıned much more easily and accurately than permeabilities Using (10) the permeability of poly-1-methylityrene in cyclohexane and toluene at iarious temperatuies and concentrations was determıned bi Mınnlieff and Jaspers ${ }^{6}$ ), some results are reproduced in table I It is remarkable that the permeability of one and the same polymer is about three tımes hıgher in the poor solvent cyclohexane than in the good solvent toluene Thw tery specific solvent effect 


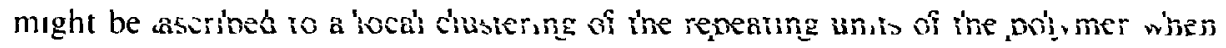
in contact with a poor solvent $A$ s a result the pores between these clusters, through which the solvent has to find its way. become wider, which leads to a steep increase in the permeability This interpretation was supported ${ }^{\circ}$ ) by the way in which the observed permea'ointies appear co úepenú on tine temperature

\section{TABLE I}

\begin{tabular}{|c|c|c|c|}
\hline \multicolumn{4}{|c|}{ 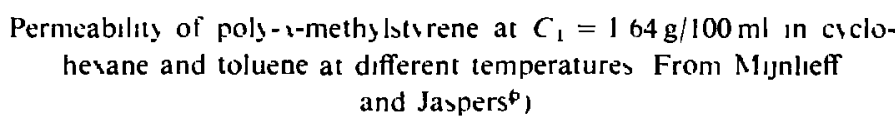 } \\
\hline $\begin{array}{l}\text { Tenip } \\
\text { ( C) }\end{array}$ & $\begin{array}{l}10^{1: 2} \\
\left(\mathrm{~cm}^{2}\right)\end{array}$ & $\begin{array}{c}\text { Temp } \\
\text { ( C) }\end{array}$ & $\begin{array}{l}10^{12} k \\
\left(\mathrm{~cm}^{2}\right)\end{array}$ \\
\hline \multicolumn{2}{|c|}{ In a. Iohe vane } & \multicolumn{2}{|c|}{ In toluene' } \\
\hline 35 & 106 & 25 & 033 \\
\hline 40 & 089 & 45 & 034 \\
\hline 50 & 070 & 65 & 036 \\
\hline 80 & 060 & 85 & 036 \\
\hline 95 & 056 & 105 & 037 \\
\hline 110 & 060 & 120 & 040 \\
\hline 125 & 061 & & \\
\hline
\end{tabular}

\section{References}

11 P Debije Phys Rer $71(19+7)+86$

2) $\mathrm{H} \mathrm{C}$ Brinkman Phrsicd $13\left(1947\right.$ 47 $^{-}$

3 B B U Felderhof and I N Deuich I Chem Phss 62 (1975) 239) 2398

4) B U Felderhof, Physica 804 11975) 63 172

5) $P$ Debije and E Huckel Phis Z 24(1923) 305

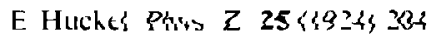

DC Hencs Pac Ra So 133 risuir ios

6) P F MIJnleft and W' I M Jaspers Tran, Far Soc 67 (1971) 1837 